\title{
Parque Verde Urbano como Espaço de Desenvolvimento Psicossocial e Sensibilização Socioambiental
}

\author{
Adria de Lima Sousa \\ Universidade Federal do Amazonas \\ Manaus, AM, Brasil \\ Josiane de Souza Medeiros \\ Faculdade Salesiana Dom Bosco \\ Manaus, AM, Brasil \\ Dayse da Silva Albuquerque \\ Universidade Federal de Santa Catarina \\ Florianópolis, SC, Brasil \\ Maria Inês Gasparetto Higuchi \\ Instituto Nacional de Pesquisas da Amazônia \\ Manaus, AM, Brasil
}

\begin{abstract}
RESUMO
Os parques verdes em grandes metrópoles têm sido identificados como espaços que promovem o bem-estar físico e mental, tendo em vista suas características que permitem ao visitante o contato com a natureza mesmo vivendo em um ambiente urbano. Esse estudo desenvolvido com visitantes de um Parque Verde Urbano (PVU) na cidade de ManausAM, investigou as motivações presentes na escolha da visitação desse espaço e os tipos de sentimentos ativados no contato com a natureza. Participaram da pesquisa 140 visitantes adultos $(\mathrm{M}=59$ e $\mathrm{F}=81)$ a partir de uma abordagem qualitativa, descritiva e exploratória com aplicação de entrevistas semiestruturadas durante sua visita ao PVU. Os resultados evidenciam que, além dos benefícios psicossociais proporcionados por esses ambientes identificados como restauradores, os visitantes vivenciaram sentimentos de valorização da natureza, despertando assim, maior sensibilização para a proteção e cuidado ambiental.
\end{abstract}

Palavras-chave: Parques Verdes Urbanos; Ambientes Restauradores; Afetividade Ambiental.

\section{ABSTRACT}

\section{Urban Green Park as Psychosocial Development Space and Socioenvironmental Awareness}

Green parks in large metropolis have been identified as spaces that promote physical and mental well-being, considering its features that allow the visitor contact with nature. This study, carried out with visitors of a Green Urban Park (GUP) in the city of Manaus-AM, investigated the motivations present in the choice of visiting this space and the types of feelings activated in contact with nature. The study was conducted with 140 adults $(\mathrm{M}=59, \mathrm{~F}=81)$ and followed a qualitative, exploratory and descriptive approach through semi-structured interviews, during their visit to the park. The results showed that, besides psychosocial benefits provided by these environments, identified as restorative, visitors experienced feelings of appreciation of nature. Such experience also aroused greater awareness of environmental protection and care among those visitors.

Keywords: Green Urban Parks; Restorative Environments; Environmental Affection.

\section{RESUMEN}

\section{Parque Verde Urbano como Espacio de Desarrollo Psicosocial y Sensibilización Socioambiental}

Los parques verdes en grandes metrópolis han sido identificados como espacios que proporcionan el bienestar físico y mental, en vista de sus características que permiten al visitante el contacto con la naturaleza. Esta investigación, realizada con visitantes de un parque verde urbano (PVU) en la ciudad de Manaus-AM, verifica que motivaciones están presentes en la elección de la visitación y los tipos de sentimientos activados en el contacto con la naturaleza. El estudio se procedió con 140 visitantes adultos $(\mathrm{M}=59, \mathrm{~F}=81)$, cuyo enfoque es cualitativo, descriptivo y exploratorio, compuesto de entrevistas semiestructuradas, mientras visitaban el parque. Los resultados mostraron que, además de los beneficios psicosociales proporcionados por estos ambientes, identificados como restauradores, los visitantes vivenciaron sentimientos de valorización de la naturaleza. Como consecuencia de ello los visitantes experimentaron una mayor conciencia de la protección y cuidado del medio ambiente.

Palabras clave: Parques Verdes Urbanos; Entornos Restauradores; Afectividad Ambiental. 


\section{INTRODUÇÃO}

As florestas, dentre outras áreas verdes, têm sido sistematicamente suprimidas com o crescimento exponencial das cidades. $\mathrm{O}$ espaço urbano acaba por se distinguir como um aglomerado de construções em detrimento da natureza, tornando-se gradativamente um ambiente insalubre física e psicologicamente. Apesar de vários estudos terem comprovado os benefícios que espaços com predominância da natureza possibilitam para o bem-estar das pessoas nas grandes cidades (Berman, Jonides, \& Kaplan, 2008; Carrus, Lafortezza, Colangelo, Dentamaro, Scopelliti, \& Sanesei, 2013; Hartig, 2004; Kaplan \& Kaplan, 1989; Ulrich, 1983), esses espaços são ainda raros nas cidades brasileiras, em especial na Amazônia. A partir das discussões relativas à preservação da natureza e da vida estressante na cidade, surge uma forte demanda social, cada vez mais crescente, da necessidade de uma maior aproximação e cuidado desses ambientes naturais. Nesse sentido, os Parques Verdes Urbanos (PVU) passam a ser uma realidade que deve estar presente no espaço citadino. Os PVU podem ser caracterizados como espaços de presença abundante de vegetação, nativa ou parcialmente plantada, que cumprem um importante papel tanto para a saúde integral das pessoas, quanto para o equilíbrio do ecossistema.

Proteger áreas naturais e fazer delas um espaço imprescindível para a vida no planeta não é recente. Diegues (2011) faz um resgate do surgimento de movimentos para criação de áreas naturais protegidas. Iniciado nos Estados Unidos, a partir de raízes ideológicas distintas, a noção de wilderness (natureza selvagem) foi base para a criação do primeiro parque natural do mundo, proveniente de ideias preservacionistas vigentes. $\mathrm{O}$ movimento ambientalista ganha força e a natureza surge como espaço a ser valorizado devido sua biodiversidade, afastando concepções ligadas à necessidade de controle da natureza pelo ser humano, ainda fortemente defendidas pela sociedade desenvolvimentista. Seguindo essa tendência, os PVU, têm se consolidado como espaços ecológicos preservados e têm se apresentado como alternativa de contato com a natureza para a busca de equilíbrio diante do estilo de vida frenético imposto pelas demandas das grandes metrópoles (Brown \& Grant, 2005; Loboda \& De Angelis, 2005; Silva, 2003; Wilson, 1999).

Espaços verdes têm sido definidos como espaços topológicos diferenciados dos espaços construídos. No senso comum bem como na literatura, ainda há falta de consenso na sua caracterização. Alguns autores consideram os PVU como espaços verdes, ou seja, áreas em que há predominância de vegetação gramínea ou arbórea (Barcellos, 1999; Castelnou, 2006; Fernandes, 2014). Esses espaços naturais inseridos em contextos predominantemente urbanos, exercem funções específicas, sejam elas ecológicas ou psicossociais.

O estudo de Steuer, Araújo, Oliveira, Silva e ElDeir (2012) identifica funções atribuídas aos PVU que se distinguem nas seguintes dimensões: (a) social - capacidade de permitir lazer e recreação, além de oportunizar novas interações sociais advindas do uso frequente do ambiente. Na dimensão social o PVU é capaz de promover errância, termo utilizado por Fischer (n.d) para referendar o ato de deslocar-se de um ambiente habitual para outro que possibilite exploração e contemplação; (b) ecológica - manutenção do microssistema, ligado à riqueza da biodiversidade e qualidade dos recursos naturais, como a água, o ar, a vegetação e sua fauna associada; (c) estética - configuração e disposição dos elementos do ambiente, com o intuito de proporcionar encantamento e fascinação aos visitantes a partir do embelezamento do espaço; (d) educativa - potencialidades para realizar atividades de educação ambiental, com o propósito de promover o cuidado e preservação dos recursos ambientais e, (e) psicológica - capacidade de proporcionar alívio das tensões diárias através do relaxamento e contemplação dos elementos naturais.

A ausência destes espaços verdes na cidade culmina em grandes perdas de benefícios para o ecossistema, como a redução da biodiversidade, a alteração no microclima e aumento da poluição sonora e do ar, a perda da qualidade do solo, a menor reciclagem de materiais e a mudança dos ciclos hidrológicos (Santos, Souza, Silva, Pinto, Lima, \& Higuchi, 2012; Souza et al., 2012). Além dos aspectos biofísicos, alguns estudos mostram que esses espaços exercem um poder restaurador às pessoas, uma vez que a natureza possui características capazes de proporcionar vivências de amplos espectros tais como bem-estar físico (Grahn \& Stigsdotter, 2010; Sugiyama, Leslie, Giles-Corti, \& Owen, 2008), social (Änggård, 2010; Cantonguay \& Jutras, 2009; Raymundo, Kuhnen, \& Soares, 2010), emocional (Corti et al., 2005; Hansman, Hug, \& Seeland, 2007) e cognitivo (Korpela, Kytia, \& Hartig, 2002; Taylor, Kuo, \& Sullivan, 2002).

Ambientes restauradores podem ser definidos como aqueles capazes de restabelecer a atenção da fadiga gerada pela concentração em atividades específicas e repetitivas a que as pessoas são submetidas em sua vida cotidiana. Para que sejam considerados restauradores, tais ambientes devem apresentar as seguintes características: 1) escape - proporcionar sensação de 
fuga por meio de distanciamento físico e psicológico; 2) fascinação - demandar atenção involuntária e interesse espontâneo; 3) escopo - oferecer uma organização que leva a um sentimento de familiaridade e pertencimento e, 4) compatibilidade - possuir uma capacidade de suprir as demandas de seus usuários (Alves, 2011; Kaplan \& Kaplan, 1989). Esses fatores não dependem da pessoa ou do ambiente isoladamente para que sejam consideradas restauradores, mas sim da interação entre ambos. As pessoas percebem tais características de acordo com suas experiências em ambientes naturais ou outros ambientes.

A necessidade do restauro psicológico (emocional e cognitivo) decorre principalmente do estilo de vida dos grandes centros urbanos que gera altos níveis de estresse e exige atenção concentrada. A literatura tem dado indicativos de que os efeitos da capacidade restaurativa do ambiente podem ser precursores de práticas de cuidado ambiental (Corral-Verdugo, 2012; Hartig, Kaiser, \& Bowler, 2001; Korpela, Ylen, Tyrvainen, \& Silvennoinen, 2010; Mayer, Frantz, BruehlmanSenecal, \& Dolliver, 2009; Nisbet, Zelenski, \& Murphy, 2011; Ryan, Weinstein, Bernstein, Brownc, Mistretta, \& Gagne, 2010). Todavia, residentes de centros urbanos podem ter sentimentos ambivalentes em relação às áreas verdes (Bonnes, Passafaro, \& Carrus, 2011). A maior parte dos estudos é desenvolvida em cidades cuja área geográfica é relativamente distante das florestas nativas. No contexto amazônico, a floresta e os fragmentos florestais apresentam-se como paisagem muito presente e próxima dos olhos, mesmo que fora do perímetro urbano, e de forma geral, não apreciada como ambiente de cidade (Higuchi, 1999; Higuchi, Azevedo, \& Forsberg, 2012; Silva, 2009). Outros estudos mostram que ambientes naturais podem induzir sentimentos negativos aos seus usuários (Willian \& Cary, 2002). Destarte tais nuances, as áreas verdes têm recebido atenção especial por desencadearem aspectos psicossociais relevantes nessa relação pessoa-ambiente.

Os sentimentos envolvidos nas relações pessoaambiente passaram a ser discutidos de maneira mais aprofundada a partir das discussões de Tuan (2012) que cunhou o termo topofilia para referendar os laços afetivos construídos pelos seres humanos com o meio ambiente material. Esses laços diferem profundamente em intensidade, sutileza e modo de expressão, de acordo com a resposta dada ao estímulo ambiental. Pode ser basicamente estética, efêmera e tátil ou mais permanente, definindo-se por meio dos significados atribuídos. A topofilia não é a emoção humana mais forte, mas, quando se apresenta dessa forma, podese concluir que o lugar é veículo de acontecimentos emocionais fortes ou é percebido como um símbolo.
A emoção afeta todas as esferas do funcionamento psicológico como uma complexa série de interações entre fatores objetivos e subjetivos e produz o aparecimento de experiências afetivas, geradoras de processos cognitivos, extensos ajustes fisiológicos e condições de estimulação que conduzem à ação. Essa ação poderá ser positiva ou negativa, pois o princípio da ação humana está relacionado a uma reação afetiva. As emoções despertadas no ambiente tanto podem trazer benefícios para saúde do usuário, como para uma ação que pressuponha o cuidado no sentido de responsabilização e zelo ambiental (Boff, 1999; Corraliza, 2002).

Ainda que haja um reconhecimento das propriedades biofísicas ou psicológicas proporcionadas pelo ambiente natural, estar nele parece ser cada vez mais difícil. Usualmente, esses lugares tornam-se preferidos para visitas em situações específicas e suas características restauradoras têm se acentuado. Em psicologia ambiental, o conceito relativo à capacidade restaurativa do ambiente tem sido uma vertente de estudo promissora, devido aos altos níveis de estresse que as pessoas têm enfrentado no cotidiano das cidades (Alves, 2011). O estresse ambiental é produzido quando as demandas do ambiente superam as capacidades de resposta das pessoas. O estilo de vida urbana tem contribuído para o estresse ambiental e um baixo nível de qualidade de vida (Evans \& Cohen, 1987; Moser \& Robin, 2006). As pessoas tendem a buscar espaços naturais a partir da percepção de suas qualidades estéticas e da possibilidade de restabelecimento e manutenção de um bem-estar físico e psicossocial (Cervinka, Röderer, \& Hefler, 2012; Howell, Dopko, Passmore, \& Buro, 2011; Lafortezza, Carrus, Sanesi, \& Davies, 2009; Perón, 2002).

Entretanto, nem sempre é possível entender todos os motivos que direcionam determinadas ações e preferências por ambientes específicos. Pesquisadores têm buscado verificar em que ambiente um turista ou visitante se sente mais confortável, qual a motivação para a escolha do local, possíveis relações com o significado que o local visitado tem para o visitante, as atividades que realiza no local de escolha, os deslocamentos efetuados na área geográfica e preferências por protótipos que correlacionam positivamente sua preferência com algo familiar como bosques e parques (Orellana, Anton, \& Viader, 2006; Perón, 2002).

Nesse sentido, alguns questionamentos conduziram o presente estudo, entre eles, quais as motivações presentes na escolha da visitação e os tipos de sentimentos ativados no contato com a natureza a partir dos PVU. O interesse por esse tema pauta-se na 
compreensão de que estudar o que as pessoas percebem e avaliam sobre espaços verdes revela-se instrutivo no que se refere à saúde integral das pessoas na cidade, à própria manutenção desses espaços como áreas de preservação e de lazer, bem como à valorização de processos educativos desenvolvidos nele.

\section{MÉTODO}

Esse estudo de caráter qualitativo com um enfoque exploratório e descritivo foi realizado por meio da aplicação de entrevistas semiestruturadas com visitantes adultos de um PVU na cidade de Manaus-AM. Como o PVU é visitado por muitos turistas estrangeiros, optamos por inserir no estudo apenas os visitantes brasileiros. A seleção dos participantes deu-se por conveniência e disponibilidade.

O PVU escolhido para realização dessa pesquisa foi o Bosque da Ciência (BC), localizado na zona centro sul da cidade. Antes de se tornar uma área de visitação, o BC era uma área de pesquisas científicas sobre o bioma amazônico, pertencente ao Instituto Nacional de Pesquisas da Amazônia (INPA). Em 1995 essa área foi transformada para se tornar um parque de visitação com a finalidade de aproximar a sociedade dos aspectos ambientais amazônicos, agregando lazer e conhecimento científico.

O BC compreende uma área de 13 hectares composta por núcleos de visitação construídos e naturais, a partir da paisagem própria daquele espaço florestal. O BC conta com trilhas planejadas entre a floresta com uma pequena mesofauna livre, aquários de mamíferos aquáticos e peixes, lagos artificiais com quelônios, répteis e abundante vegetação nativa, além de um centro de exposição de curiosidades da fauna e flora amazônica. Esse conjunto paisagístico foi planejado para disponibilizar aos visitantes elementos estéticos (percepcionais, emocionais e afetivos), biofísicos (relacionados à diversidade, biodiversidade $\mathrm{e}$ integridade natural) e elementos culturais da paisagem, aspectos históricos e socioeconômicos presentes na região (Saraiva \& Lavrador-Silva, 2005).

\section{Participantes, procedimentos e instrumentos}

Foram entrevistados 140 visitantes $(\mathrm{M}=59$ e $\mathrm{F}=81)$ com idade entre 18 e 77 anos e com diferentes níveis de escolaridade: ensino fundamental (4\%); ensino médio (30\%); ensino superior incompleto $(22 \%)$ e ensino superior completo (44\%). A maioria dos visitantes entrevistados (51\%) residia na cidade de Manaus, 5\% eram do interior do Amazonas e 44\% de outras cidades e regiões do país.
Esses visitantes foram entrevistados a partir de um roteiro de perguntas abertas. As entrevistas, com duração média de 15 minutos, foram realizadas em dias diferenciados da semana (terça a domingo - dias de funcionamento do PVU), nos períodos da manhã e tarde, logo após o término da visita. As respostas dadas pelos participantes foram audiogravadas e complementadas com informações demográficas registradas no próprio protocolo de pesquisa para posterior análise.

A pesquisa desenvolvida seguiu os trâmites de procedimento ético da RE 196-MS, sendo pois, submetida e aprovada pelo Comitê de Ética em Pesquisa com Seres Humanos do Instituto Nacional de Pesquisas da Amazônia (CEP/INPA), sob protocolos de $\mathrm{n}$ o 043-10 e 025-11. A pesquisa aqui apresentada teve o apoio do Conselho Nacional de Desenvolvimento Científico e Tecnológico (CNPq) e da Fundação de Amparo à Pesquisa do Amazonas (FAPEAM) através de bolsas de iniciação científica.

Os procedimentos para análise de dados das entrevistas consistiram inicialmente na criação de uma planilha onde foram inseridos dados sociodemográficos e a transcrição na íntegra das repostas dadas pelos entrevistados. Os dados foram submetidos à análise de conteúdo a partir do método de Bardin (2011), o qual consiste em um conjunto de técnicas de análise das comunicações. A partir dessa análise emergiram categorias que expressam as motivações da visita ao PVU, sentimentos vivenciados, preferências acerca da paisagem e a importância atribuída aos elementos ambientais observados. As categorias foram validadas por dois pesquisadores da área, com no mínimo $70 \%$ de coerência. Com a elucidação das categorias relativas à motivação e sentimentos foram elaboradas estatísticas descritivas para verificar o percentual de participantes que evocaram cada respectiva categoria de pensamento.

\section{RESULTADOS E DISCUSSÃO}

\section{Motivações para visitação do Parque Verde Urbano}

Considera-se que o fato de alguém decidir por visitar um determinado lugar esteja atrelado a uma motivação. A motivação relaciona-se com a predisposição para direcionar comportamentos pautados em objetivos que possibilitam a permanência ou transformação de determinada situação (Braghrirolli, Bisi, Rizzon, \& Nicoletto, 2009). Ao analisar o conteúdo latente das motivações que levaram os entrevistados à visita do referido PVU, constataramse quatro categorias distintas: (a) curiosidade sobre a fauna e flora amazônica; (b) efeito restaurativo do 
PVU; (c) prática do turismo e lazer e, (d) contato com a natureza.

Observou-se que a motivação acionada pela curiosidade sobre a fauna e flora foi expressiva para $44 \%$ dos entrevistados. O interesse em conhecer animais e plantas amazônicas e os respectivos habitats no bioma amazônico os fizeram se sentir motivados para visitar o BC como PVU que contém tais elementos. Pelo fato do BC pertencer ao INPA, e este ser um instituto de pesquisas científicas, a curiosidade relativa aos estudos realizados pelos cientistas à sociedade foi saliente: " $O$ principal motivo mesmo era conhecer o INPA, eu ouço falar muito do INPA lá no Rio" (F, 23 anos). A estreita relação do INPA com o ambiente amazônico e o fato do $\mathrm{BC}$ representar um fragmento da região motivou o visitante a "saber mais sobre a Amazônia, os animais, as plantas... ver de perto a Amazônia. Eu sempre quis vir aqui." (M, 56 anos).

Um dos principais fatores que contribui para a existência da apreciação positiva com um determinado lugar trata-se do interesse de conhecer os elementos constituintes de um espaço (Tuan, 2012). Nesse sentido, o BC é apresentado na mídia local como um lugar onde se pode ver alguns espécimes amazônicos bastante atrativos (www.inpa.gov.br). Entre os animais que se destacam está o peixe-boi, mamífero aquático ameaçado de extinção e estudado pelos pesquisadores do INPA. O grande aquário de peixes-boi expõe filhotes e adultos para serem apreciados pelos visitantes. O peixe-boi de água doce faz parte do imaginário popular por ser pouco acessível para aqueles que nunca adentraram os rios da Amazônia, por isso "Sempre tive vontade de vir vê o peixe-boi e mostrar os animais para minhas filhas" (M, 28 anos).

Para 25\% dos entrevistados a motivação para a visita ao PVU foi a busca de um bem-estar proporcionado pelo contato com a natureza. Esse contato com a mata e animais dão, segundo esses entrevistados, a tranquilidade necessária para enfrentar o dia-a-dia estressante e agitado da cidade. O PVU com suas características naturais e relativa tranquilidade permite restauro psicológico para “... desestressar um pouco. Ultimamente ando muito estressado" (M, 25 anos); ou "pra esquecer o dia-a-dia infernal da cidade, pra fugir do tormento da cidade, tirar o estresse" (M, 62 anos). Observa-se que o ambiente natural, com uma paisagem com plantas, animais, trilhas entre árvores e espelhos d'água lhes serve como contraponto ao barulho e agitação urbana. Nesse espaço, o sujeito se encontra diante da possibilidade de um equilíbrio das emoções, justamente pela característica distintiva que a natureza proporciona. Nota-se que esse estado já parece ter sido vivenciado pelo sujeito e, portanto se consolida como uma opção de espaço a ser frequentado. Para esses entrevistados, o ambiente do PVU se constitui cognitiva e afetivamente restaurador. A capacidade do ambiente natural presente no PVU já está incorporada como coadjuvante do restauro psicológico. É nessa paisagem, contrastante com a intensidade da urbanização frenética, que os citadinos encontram a calma e tranquilidade para recuperarem ou renovarem suas emoções e condicionamento físico. Nesse sentido, o PVU já é visto como um recurso ambiental capaz de devolver a capacidade cognitiva e funcional para enfrentar a vida na cidade. Esse resultado corrobora com outros estudos no reconhecimento do PVU como um ambiente que oferece estímulos potencialmente restauradores (Hartig, 2011; Ribeiro, 2008).

O PVU também é percebido como instância recreadora para $22 \%$ dos entrevistados. É nesse espaço, com predomínio da natureza, que os entrevistados percebem o lugar ideal para passear e mostrar aos amigos, namorados e familiares as belezas da região amazônica. Nesse ponto, é possível distinguir a identidade de lugar associada ao ambiente físico, que de alguma forma retrata o sentido do ser amazônico, aquele que vive na pele a alta umidade e temperatura numa floresta típica dos trópicos, com animais e plantas que só ali tem. O PVU aproxima a floresta longínqua, que mesmo estando nela, não se pode nela chegar. O PVU na Amazônia encurta o caminho com os mesmos atrativos da flora e fauna da mata virgem, porém com a segurança necessária. É um passeio seguro, que sugere turismo e passeio e assim poder "apresentar a floresta amazônica para meu namorado que é de Florianópolis" (F, 34 anos); e divertir-se "pelo lazer ao ver os animais" (M, 23 anos); e "estar passeando com a família” (F, 32 anos). Perceber uma área verde como espaço de lazer não é novidade mundo afora. Vários estudos trazem essa confirmação no sentido de reconhecer uma área demarcada como um espaço que transcende sua condição física com seus recursos naturais a serem preservados, mas também um espaço social, destinado à recreação (Silva, 2003), realização de atividades físicas (Duvall, 2011; Thompson, 2013), expansão das redes sociais (Steuer et al., 2012) e errância (Fischer, n.d.).

Uma visita para ter contato com a natureza é outro motivo para estar num PVU para 12\% dos entrevistados. Embora tenha sido a categoria de entendimento com menor percentual de expressão, o contato direto e íntimo com a floresta, lagos e animais proporciona, na visão destes visitantes, uma compensação pelo distanciamento da natureza. A aproximação se dá por meio do PVU e permite a esses entrevistados adultos "mostrar para os filhos a importância de cuidar dos 
animais e da floresta" (M, 50 anos); e ao mesmo tempo "sensibilizar o filho sobre a importância de preservar os animais" (F, 46 anos). Desse modo, o PVU se torna um espaço de mudança do comportamento ambiental, onde os visitantes percebem que há emergência e urgência na proteção e preservação da biodiversidade contida na Amazônia. A imersão na floresta possibilita um contato intenso e íntimo capaz de transformar práticas em prol da conservação da biodiversidade, que esses pais vislumbram para seus filhos seja na infância ou juventude (Higuchi, Azevedo, \& Forsberg, 2012).

A participação dos jovens em diferentes espaços sociais tem sido apontada como fator importante para a formação de indivíduos críticos e com a capacidade de refletir sua própria prática social em uma perspectiva ética e ecológica (Castro, 2009; Higuchi, 2008; Janke \& Reis, 2008). É importante destacar que para esses entrevistados, a nova geração teria muitos ganhos visitando PVUs, entre eles, "Diria que é importante pelas pesquisas, pra mudar o ambiente que os adolescentes frequentam, conhecer coisas novas" (M, 23 anos). Ou ainda "Diria pra experimentar o contato com a natureza, os animais... Traz um prazer para nós, porque a gente foge da rotina, ao mesmo tempo em que você tem o conhecimento da natureza você adquire mais conhecimento sobre ela" (F, 30 anos).

Essas experiências e conhecimentos estão associados com a qualidade e cuidado do ambiente natural, isto é, aos aspectos de arranjo espacial e da paisagem próprias do lugar. Esses resultados dialogam com outros trabalhos (Berto, Baroni, Zainaghi, \& Betella, 2010; Korpela et al., 2010; Tyrvainen et al., 2014), no que se refere à compreensão dos parques naturais urbanos como elementos indispensáveis para a homeostase ambiental; bem como com os que destacam as relações sociais nas grandes metrópoles que têm nos PVU um alento (Francis, Giles-Corti, Wood, \& Knuiman, 2012; Korpela, Borodulin, Neuvonen, Paronen, \& Tyrvainen, 2014; Zhang, Piff, Iyer, Koleva, \& Keltner, 2014).

As motivações aqui apresentadas remetem ao fato de que tanto as características do lugar quanto as demandas próprias da pessoa impulsionam o interesse no PVU. Por um lado, a motivação está associada às informações e condições próprias do lugar. Por outro lado, emoções em desalinho pelo estresse diário motivam a procura do PVU como alvo espacial para o restauro psicológico. A natureza nele presente e seus efeitos restauradores são preponderantes para a preferência em detrimento a outros espaços (Alves, 2011; Kaplan \& Kaplan, 1989; Ulrich, 1991). Além disso, o PVU possibilita ainda reavivar os ideais preservacionistas diante da necessidade de proteção da natureza. O PVU pode se constituir um espaço de aliança para com a natureza que a sociedade tratou de afastá-la e ignorá-la como aspecto coadjuvante da vida planetária. Portanto, novas socialidades podem surgir a partir da espacialidade dos PVU (Fischer, n.d.).

As motivações encontradas nesse estudo para as pessoas visitarem um PVU, podem nos auxiliar tanto no redirecionamento e organização desses espaços na cidade, como valorizar e reconhecer nas características de ambiente natural. As possibilidades de melhorias na qualidade de vida e saudabilidade das pessoas, de modo especial aos citadinos, que sofrem as influências da aglomeração e agitação das grandes cidades, passam a ser reconhecidas (Galindo, Gilmartín, \& Corraliza, 2002).

Observa-se, no entanto, a necessidade de mais estudos sobre essa temática no Brasil, conforme defendem Alves e Betrabet-Guwaldi (2008) e Gressler e Günther (2013), pois ainda pouco conhecimento se tem a respeito da influência dos aspectos socioculturais na percepção e uso de ambientes.

\section{Sentimentos vivenciados na visitação de Parques Verdes Urbanos}

Sabe-se que os diferentes lugares possuem características capazes de gerar diversos sentimentos (Corraliza, 2002; Bomfim, 2010). Esses sentimentos estão, em última instância, associados às emoções que levam à ação e estes podem ter valências negativas ou positivas. Ao estarem em um ambiente como o PVU, os entrevistados relataram que vivenciaram sentimentos específicos. A grande maioria (88\%) destacou sentimentos que envolvem características de restauro psicológico e uma pequena parcela (9\%) atribuiu sentimentos relacionados ao cuidado ambiental. Apenas 3\% não souberam precisar o tipo de sentimento vivenciado na visita ao PVU.

Os sentimentos próprios de restauro psicológico proporcionados pelo ambiente do PVU foram expressos de forma complementar. Por um lado escape, fuga da rotina, alívio, relaxamento, escopo, contemplação, tranquilidade e calma, em oposição ao estresse do dia-a-dia. "É uma paz... Uma tranquilidade e ajuda a desestressar também do cotidiano do dia-a-dia" (M, 28 anos); "Eu venho aqui porque gosto de vir por isso, porque a gente vem aqui e relaxa. Sente até uma paz por causa do ambiente, dos animais, da natureza... as florestas" (F, 67 anos).

Por outro lado, sentimentos relativos ao estar bem pelo fato dessa presença que permite o surgimento de alegria, felicidade e amor ao estar em contato com a natureza. Ao ver animais nunca vistos antes, sentir tranquilidade e sossego favoreceu um reencontro 
com lembranças de momentos felizes de outras épocas da vida. $\mathrm{O}$ encantamento ao entrar em contato com a natureza mostra que esta é capaz de surpreender quem vive na cidade "Fiquei impressionado e feliz, a gente é de cidade grande e dificilmente a gente vê esse tipo de coisa" (M, 50 anos).

Observa-se que a aproximação com a natureza desperta sentimentos prazerosos nas pessoas, reforçando a ideia de que o ser humano não é uma instância separada ou polarizada do ambiente. Ao contrário, as múltiplas dimensões do espaço constituem-se por sua interação com as subjetividades construídas pelas pessoas em suas vivências. Assim, os espaços são afetados pelas intersubjetividades e estas por eles ao mesmo tempo (Bertini, 2007; Bomfim, 2010).

Nesta direção, sentimentos relacionados ao cuidado ambiental foram ativados num pequeno grupo durante a visita incluindo preocupação, tristeza e pesar diante da possibilidade de perda da natureza presente no PVU. Tal sensibilidade, que transcende a cronologia presente, leva a um sentimento de necessidade de cuidado, pois "a gente aprende a valorizar um pouco né... A gente pensa como as pessoas conseguem destruir uma coisa tão linda... Quando vejo essa destruição me dá um pouco de tristeza" (F, 22 anos). É interessante notar como esse sentimento parece se relacionar com a esfera da ética do cuidado, no sentido de se responsabilizar por algo, se preocupar e querer zelar por esses ambientes e os seres que neles habitam (Boff, 1999).

Observa-se que esse estudo nos indica, juntamente com outros tantos, que o ambiente é capaz de despertar sentimentos diversos, sobretudo positivos que se relacionam com memórias e informações diversas, que são evocados quando em contato direto (Corral-Verdugo, 2012). Naturalmente, as pessoas se distinguem em seus laços afetivos diante do meio ambiente material.

A preferência por ambientes com predomínio de natureza é destacada por pesquisadores que se propõem a discutir a Teoria da Restauração da Atenção (ART), resultante dos estudos de Kaplan e Kaplan (Beute \& Kort, 2013; Galindo, Gilmartin \& Corraliza; 2002). A conotação relativa a ambientes naturais que não comportam artefatos humanos pode distinguir-se dos ambientes construídos, e essa distinção deve-se as percepções, intenções e expectativas dos indivíduos, na medida em que eles se ligam a atividades nos dois tipos de paisagem (Barracho, 2001; Moser, 2006).

\section{CONSIDERAÇÕES FINAIS}

Os resultados obtidos neste estudo nos indicam que os PVU podem ser discutidos a partir de um entendimento deste como um espaço de desenvolvimento psicossocial e ainda como um disparador para sensibilização quanto às questões socioambientais. Os aspectos do restauro psicológico, percebidos a partir dos benefícios emocionais e cognitivos associados a esses ambientes, revela a importância de paisagens com elementos naturais e os benefícios que estas trazem para a saúde e bem-estar.

Embora os estudos tenham evidenciado que há uma relação com a apreciação do PVU a partir dos aspectos restauradores, esse fator se revela como motivação principal para poucos quando na escolha da visitação desses espaços. Isso pode indicar uma necessidade de maiores investigações sobre essa relação em contextos socioambientais distintos, tendo em vista as especificidades de cada região, sobretudo numa região como a amazônica onde a exuberância da natureza é encontrada muito próxima da cidade.

Os espaços naturais demarcados como PVU podem trazer aos indivíduos uma possibilidade de restabelecimento tanto da cidadania ambiental quanto da responsabilidade social. A possibilidade de suscitar nos visitantes uma reflexão que sugere o cuidado ambiental envolve sentimentos articulados com as características restauradoras do ambiente.

A aproximação da sociedade em relação aos PVU pressupõe um envolvimento maior em atividades de cunho socioambiental e um desenvolvimento psicossocial mais sadio, em decorrência dos sentimentos despertados pelo ambiente e pelo contato constante com estímulos que despertam sensações prazerosas. Esse panorama condiz com o afeto ético do cuidado, presente no discurso de visitantes de ambientes naturais. Admite-se, por fim, que a transformação de PVU em espaço de aprendizagem e envolvimento social constitui-se numa proposta de construção de novos modos de sentir, pensar e agir as/nas relações pessoa-ambiente.

Considerando as limitações deste estudo sugerese, por fim, que novos estudos sejam realizados para maior aprofundamento das relações previas desses entrevistados com outros ambientes e dinâmicas próprias de seus estilos de vida. 


\section{REFERÊNCIAS}

Alves, S. M. (2011). Ambientes Restauradores. In S. Cavalcante \& G.A. Elali (Org.). Temas Básicos em Psicologia Ambiental (pp. 44-52). São Paulo: Editora Vozes.

Alves, S.M. \& Betrabet-Guwaldi, G. (2008). Interação humana com ambientes naturais: uma revisão no periódico Environment and Behavior. In J.Q. Pinheiro \& H. Günther (Org.). Métodos de Pesquisa nos Estudos Pessoa-Ambiente (pp. 343-368). São Paulo: Casa do Psicólogo.

Änggård, E. (2010). Making Use of "Nature" in an Outdoor Preschool: classroom, home and fairyland. Children, Youth and Environments, 20(1), 4-25.

Bardin, L. (2011). Análise de Conteúdo. São Paulo: Edições 70.

Barcellos, V. Q. (1999). Os Parques como espaços livres públicos de lazer: o caso de Brasília. Tese. FAU/USP, São Paulo.

Barracho, C. (2001). Psicologia Social: Ambiente e Espaço (2ª ed.). Lisboa: Instituto Piaget.

Berman, M.G., Jonides, J., \& Kaplan, S. (2008). The cognitive benefits of interacting with nature. Psychological Science, 19(12), 1207-1212. http://dx.doi:10.1111/j.1467-9280.2008.02225.x

Bertini, F. (2007). Centro de Fortaleza entre afetos e sentidos. Fortaleza: FATECI.

Berto, R., Baroni, M. R., Zainaghi, A., \& Bettella, S. (2010). An exploratory study of the effect of high and low fascination environments on attentional fatigue. Journal of Environmental Psychology, 30(4), 494-500. http://dx.doi:10.1016/j. jenvp.2009.12.002

Beute, F. \& Kort, Y.A.W. (2013). Let the sun shine!: measuring explicit and implicit preference for environments differing in naturalness, weather type and brightness. Journal of Environmental Psychology, 36, 162-178. http:// dx.doi:10.1016/j.jenvp.2013.07.016

Boff, L. (1999). Saber Cuidar: Ética Humana - compaixão pela terra. Petrópolis: Vozes.

Bomfim, Z. A. C. (2010). Cidade e Afetividade: estima e construção de mapas afetivos de Barcelona e de São Paulo. Fortaleza: Editora da UFC.

Bonnes, M., Passafaro, P., \& Carrus, G. (2011). The ambivalence of atitudes toward urban green areas: Between proenvironmental worldviews and daily residential experience. Environment and Behavior, 43, 207-232. http:// dx.doi:10.1177/0013916509354699

Braghirolli, E. M., Bisi, G. P., Rizzon, L. A., \& Nicoletto, U. (2009). Psicologia Geral (28a ed.). Petrópolis: Vozes.

Brown, C., \& Grant, M. (2005). Biodiversity and human health: What role for nature in healthy urban planning? Built Environment, 31(4), 326-338. ISSN 0263-7960.

Carrus, G., Lafortezza, R., Colangelo, G., Dentamaro, I., Scopelliti, M., \& Sanesi, G. (2013). Relations between naturalness and perceveid restorativeness of different urban green spaces. Psyecology, 4, 227-244. http://dx.doi: $10.1174 / 217119713807749869$

Castelnou, A. M. N. (2006). Parques Urbanos de Curitiba: De espaços de lazer a objetos de consumo. Cadernos de Arquitetura e Urbanismo, Belo Horizonte, 13(14), 53-73.

Castonguay, G. \& Jutras, S. (2009). Children's appreciation of outdoor places in a poor neightborhood. Children, Youth and Environments, 20(1), 200-230.

Castro, L. R. (2009). Juventude e socialização política: atualizando o debate. Psicologia: Teoria e Pesquisa, 25(4), 479487. http://dx.doi:10.1590/S0102-37722009000400003

Cervinka, R., Röderer, K., \& Hefler, E. (2012). Are nature lovers happy? On various indicators of well-being and connectedness with nature. Journal of Health Psychology, 17, 379-388. http://dx.doi.org/10.1177/1359105311416873

Corraliza, J. A. (2002). Emoción y Ambiente. In J.I. Aragonés, \& M. Amérigo (Coord.). Psicologia Ambiental (pp. 59-76). Madri: Ediciones Pirámide.

Corral-Verdugo, V. (2012). Sustentabilidad y Psicologia Positiva: uma visión optmista de las conductas proambientales y prosociales. Mexico: Editorial el Manual Moderno.

Corti, B. G., Broomhall, M. H., Knuiman, M., Collins, C., Douglas, K., Ng, K., Lange, A., \& Donovan, R. J. (2005). How important is Distance to, attractiveness, and size of public open space? American Journal of Preventive Medicine, 28, 169-176.

Diegues, C.A. (2011). O Mito Moderno da Natureza Intocada (3ª ed.). São Paulo: HUCITEC.

Duvall, J. (2011). Enhancing the benefits of outdoor walking with cognitive engagement strategies. Journal of Environmental Psychology, 31(1), 27-35. http://dx.doi:10.1016/j.jenvp.2010.09.003

Evans, G. W. \& Cohen, S. (1987). Environmental Stress. In D. Stokols \& I, Altman (Ed.). Handbook of Environmental Psychology (pp. 571-610). John Wiley \& Sons, New York.

Grahn, P. \& Stigsdotter, U. K. (2010). The relation between perceived sensory dimensions of urban green space and stress restoration. Landscape and Urban Planning, 94, 264-275. http://dx.doi.org/10.1016/j.landurbplan.2009.10.012

Fernandes, K. M. (2014). Gestão e uso de Parques Verdes Urbanos como cenários sociais de proteção ambiental. Dissertação de Mestrado. Universidade Federal do Amazonas, Manaus.

Fischer, G. (n.d.). Psicologia Social do Ambiente. Lisboa: Instituto Piaget.

Francis, J., Gilles-Corti, B., Wood, L., \& Knuiman, M. (2012). Creating sense of community: The role of public space. Journal of Environmental Psychology, 32, 401-409. http://dx.doi:10.1016/j.jenvp.2012.07.002 
Galindo, M. P., Gilmartín, A. G., \& Corraliza, J. A. (2002). El médio natural. In J. I. Aragonés \& M. Amérigo (Coord.). Psicologia Ambiental (pp. 281-307). Madri: Ediciones Pirámide.

Gressler, S.C., \& Gunther, I. A. (2013). Ambientes Restauradores: definição, histórico, abordagem e pesquisas. Estudos de Psicologia, 18(3), 487-495. http://dx.doi:10.1590/S1413-294X2013000300009

Hansmann, R., Hug, S. M., \& Seeland, K. (2007). Restoration and stress relief through physical activities in forests and parks. Urban Forestry \& Urban Greening, 6, 213-225. http://dx.doi.org/10.1016/j.ufug.2007.08.004

Hartig, T. (2004). Restorative Environments. In C. Spielberger (Ed.), Encyclopedia of applied psychology (Vol. 3) (pp. 273-278). San Diego: Elsevier/Academic Press.

Hartig, T. (2011). Issues in restorative environments research: matters of measurement. In B. Fernandez-Ramírez, C.H. Villodres, C.M.S. Ferrer, \& M.J.M. Méndez (Ed.), Psicología Ambiental 2011: entre los studios urbanos y el análisis de la sostenibilidad. (pp. 41-66). Universidad de Almería: Associación de Psicologia Ambiental (PSICAMB). ISBN: 978-84-693-9260-7.

Hartig, T., Kaiser, F. G., \& Bowler, P. A. (2001). Psychological Restoration as a positive motivation for ecological behavior. Environment and Behavior, 33(4), 590-607. http://dx.doi:10.1177/00139160121973142

Higuchi, M. I. G. (1999). House, Street, Bairro and Mata: Ideas of Place and Space in an Urban Location in Brazil. Tese. Brunel University, Inglaterra.

Higuchi, M. I. G. (2008). Construindo caminhos de protagonismo socioambiental com adolescentes. In L. R. Castro \& V. L. Besset (Org.). Pesquisa-Intervenção na infância e juventude (pp. 224-243). Rio de Janeiro: FAPERJ/Nau Editora.

Higuchi, M. I. G., Azevedo, G. C., \& Forsberg, S. S. (2012) A floresta e sociedade: ideias e práticas históricas. In M.I.G. Higuchi, \& N. Higuchi (Ed.), A floresta amazônica e suas múltiplas dimensões: uma proposta de educação ambiental (2ª ed. rev. ampl.) (pp. 311-329). Manaus.

Howell, A. J., Dopko, R. L., Passmore, H., \& Buro, K. (2011). Nature connectedness: Associations with well-being and mindfulness. Personality and Individual Differences, 51, 166-171. http://dx.doi.org/10.1016/j.paid.2011.03.037

Janke, N. \& Reis, M. F. C. (2008). Produção Coletiva de Conhecimento sobre Qualidade de Vida: Por uma Educação Ambiental participativa e emancipatória. Ciência e Educação, 14. http://dx.doi.org/10.1590/s1516-73132008000100010

Kaplan, R. \& Kaplan, S. (1989). The experience of nature: A psychological perspective. Cambridge University Press.

Korpela, K., Borodulin, K., Neuvonen, M., Paronen, O., \& Tyrvainen, L. (2014). Analyzing the mediators between nature-based outdoor recreation and emotional well-being. Journal of Environmental Psychology, 37, 1-7. http:// dx.doi:10.1016/j.jenvp.2013.11.003

Korpela, K., Kytia, M., \& Hartig, T. (2002). Restorative experience, self regulation, and children's place preferences. Journal of Environmental Psychology, 22, 338-398. http://dx.doi.org/10.1006/jevp.2002.0277

Korpela, K.M., Ylen, M., Tyrvainen, L., \& Silvennoinen, H. (2010). Favorite Green, Waterside and Urban Environments, Restorative Experiences and Perceived Health in Finland. Health Promotion International, 25(2), 200-209. http:// dx.doi:10.1093/heapro/daq007

Lafortezza, R., Carrus, G., Sanesi, G., \& Davies C. (2009). Benefits and well-being perceived by people visiting green spaces in periods of heat stress. Urban Forestry \& Urban Greening, 8(2), 97-108. http://dx.doi.org/10.1016/j. ufug.2009.02.003

Loboda, C. R. \& De Angelis, B. L. D. (2005). Áreas verdes públicas urbanas: conceitos, usos e funções. Revista do Centro de Ciências Agrárias e Ambientais, 1(1), 129-139. Recuperado em 18 jan. 2011, de http://revistas.unicentro. br/index.php/ambiencia/article/view/157/185

Mayer, S. F., Frantz, C. M., Bruehlman-Senecal, E., \& Dolliver, K. (2009). Why is nature beneficial? The role of connectedness to nature. Environment and Behavior, 41, 607-643. http://dx.doi.org/10.1177/0013916508319745

Moser, G., \& Robin, M. (2006). Environmental annoyances: an urban-specific threat to quality of life? Revue Européene de Psychologie Appliquée, 56(1), 35-41. http://dx.doi:10.1016/j.erap.2005.02.010

Nisbet, E. K., Zelenski, J. M., \& Murphy, S. A. (2011). Happiness is in our nature: Exploring nature relatedness as a contributor to subjective well-being. Journal of Happiness Studies, 12, 303-322. http://dx.doi.org/10.1007/s10902010-9197-7

Orellana, A., Anton, S., \& Viader, T. (2006). La relación con el entorno como variable de segmentación de turistas en destinos litorales. In J. A. Corraliza, J. Berenguer, \& R. Martín (Ed.). Medio Ambiente, Bienestar Humano y Responsabilidade Ecológica. Madrid: Universidad Autónoma de Madrid.

Perón, E. (2002). Environmental preferences and restorative capacity of places. In R. G. Mira, J. M. Cameselle, \& J. R. Martinez (Ed.). Psicologia y Médio Ambiente: Aspectos Psicosociales, Educativos y Metodológicos (pp. 263-276). Coru-a: IMAGRAF.

Raymundo, L. S., Kuhnen, A. \& Soares, L. (2010). O espaço aberto da educação infantil: lugar para brincar e desenvolver-se. Psicologia em Revista, 16(2), 251-270.

Ribeiro, R. D. (2008). Influência da percepção de segurança no restauro cognitivo em ambientes urbanos e naturais. Dissertação de Mestrado. Universidade de Lisboa a Faculdade de Psicologia e de Ciências.

Ryan, R. M., Weinstein, N., Bernstein, J., Brownc., K. W., Mistretta, L., \& Gagne, M. (2010). Vitalizing Effect of Being Outdoors and in Nature. Journal of Environmental Psychology, 30(2), 159-168. http://dx.doi.org/10.1016/j. jenvp.2009.10.009 
Santos, J., Souza, C.A.S., Silva, R.P., Pinto, A.C.M., Lima, A.J.N., \& Higuchi, N. (2012). Amazônia: características e potencialidades. In M.I.G. Higuchi \& N. Higuchi (Ed.). A floresta amazônica e suas múltiplas dimensões: uma proposta de educação ambiental (2ª ed. rev. amp.). Manaus: Edição dos autores.

Saraiva, M. G. \& Labrador-Silva, A. (2005). Percepção e Avaliação dos valores estéticos da paisagem. Síntese metodológica. In Socza (Org.). Contextos Humanos e Psicologia Ambiental (pp. 382-385). Lisboa: Fundação Calouste Gulbenkian.

Silva, M. P. S. C. (2009). Aqui é melhor do que lá: Representação Social da vida urbana das populações migrantes e seus impactos socioambientais em Manaus. Manaus: Editora da Universidade Federal do Amazonas.

Silva, L. D. J. M. D. (2003). Parques Urbanos: A Natureza na Cidade-uma análise da percepção dos atores urbanos. Dissertação de Mestrado, Universidade de Brasília. Centro de Desenvolvimento Sustentável, Brasília/DF.

Souza, C.A.S., Silva, R.P., Barros, P., Durgante, F.M., Amaral, M.R.M., Silva, N.C., Santos, J.S., \& Higuchi, N. (2012). Floresta amazônica: Conceitos fundamentais. In M.I.G. Higuchi, \& N. Higuchi (Ed.) A floresta amazônica e suas múltiplas dimensões: uma proposta de educação ambiental (2ª ed. rev. amp.). Manaus: Edição dos autores.

Steuer, I. R. W., Araújo, G.V.R., Oliveira, B. M. C., Silva, T. E. P., \& El-Deir, S. G. (2012). Gerenciamento de Áreas Verdes na Universidade Federal Rural de Pernambuco (UFRPE) para recomposição florestal. Anais do III Congresso Brasileiro de Gestão Ambiental.

Sugiyama, T., Leslie, E., Giles-Corti, B., \& Owen, N. (2008). Associations of neighborhood greenness with physical and mental health: do walking, social coherence and local social interaction explain the relationships? Journal of Epidemiology and Community Health, 62, 9-15. http://dx.doi.org/10.1136/jech.2007.064287

Taylor, A. F., Kuo, F. E., \& Sullivan, W. C. (2002). Views of nature and self-discipline: evidence from inner city children. Journal of Environmental Psychology, 22, 49-63. http://dx.doi.org/10.1006/jevp.2001.0241

Thompson, C. W. (2013). Activity, exercise and the planning and design of outdoor spaces. Journal of Environmental Psychology, 34, 79-96. http://dx.doi:10.1016/j.jenvp.2013.01.003

Tuan. T. (2012). Topofilia: Um estudo da percepção, atitudes e valores do meio ambiente. Londrina: Eduel.

Ulrich, R. S. (1983). Aesthetic and affective response to natural environment. In I. Altman, \& J. F. Wohlwill (Ed.). Behaviour and the natural environment (pp. 85-125). New York: Plenum Press.

Ulrich, R. S. et al. (1991). Stress Recovery During Exposure to Natural and Urban Environments. Journal of Environmental Psychology, 11, 201-230. http://dx.doi.org/10.1016/S0272-4944(05)80184-7

Williams, K. J. H., \& Cary, J. (2002). Landscape preferences, ecological quality, and biodiversity protection. Environment and Behavior, 34(2), 257-274. http://dx.doi:10.1177/0013916502034002006

Wilson, E. O. (1999). The diversity of life. New York: W. W. Norton.

Zhang, J.W., Piff, P.K., Iyer, R., Koleva, S., \& Keltner, D. (2014). An ocasion for unselfing: Beautiful nature leads to prosociality. Journal of Environmental Psychology, 37, 61-72. http://dx.doi:10.1016/j.jenvp.2013.11.008

\author{
Autores: \\ ADRIA DE LIMA SOUSA - Universidade Federal do Amazonas. \\ Josiane de Souza MedeIros - Faculdade Salesiana Dom Bosco. \\ DAYSE DA Silva AlbuQuerQue - Universidade Federal de Santa Catarina. \\ MARIA INÊS GASPARETTO HigUCHI - Instituto Nacional de Pesquisas da Amazônia. \\ Endereço para correspondência: \\ Maria Inês Gasparetto Higuchi \\ Av. André Araujo, 2936 - Petrópolis \\ CEP 69067-375 Manaus, AM, Brasil \\ Recebido em: 28.05 .14 \\ Aceito em: 01.06.15
}

